\title{
Ground-state isolation and discrete flows in a rationally extended quantum harmonic oscillator
}

\author{
José F. Cariñena ${ }^{1, *}$ and Mikhail S. Plyushchay ${ }^{2, \dagger}$ \\ ${ }^{1}$ Departamento de Física Teórica, Universidad de Zaragoza, 50009 Zaragoza, Spain \\ ${ }^{2}$ Departamento de Física, Universidad de Santiago de Chile, Casilla 307, Santiago 2, Chile
}

(Received 19 September 2016; published 23 November 2016)

\begin{abstract}
Ladder operators for the simplest version of a rationally extended quantum harmonic oscillator (REQHO) are constructed by applying a Darboux transformation to the quantum harmonic oscillator system. It is shown that the physical spectrum of the REQHO carries a direct sum of a trivial and an infinitedimensional irreducible representation of the polynomially deformed bosonized $\mathfrak{o} \mathfrak{s} \mathfrak{p}(1 \mid 2)$ superalgebra. In correspondence with this the ground state of the system is isolated from other physical states but can be reached by ladder operators via nonphysical energy eigenstates, which belong to either an infinite chain of similar eigenstates or to the chains with generalized Jordan states. We show that the discrete chains of the states generated by ladder operators and associated with physical energy levels include six basic generalized Jordan states, in comparison with the two basic Jordan states entering in analogous discrete chains for the quantum harmonic oscillator.
\end{abstract}

DOI: 10.1103/PhysRevD.94.105022

\section{INTRODUCTION}

Darboux transformations, introduced originally as a method to solve linear differential equations and generalized subsequently for Darboux-Crum(-Krein-Adler) transformations [1-5], find many important applications in physics. For a long period of time they were used in quantum mechanics in the factorization method for solving the Schrödinger equation [6-9]. Nowadays they are exploited intensively in the context of supersymmetry. These transformations lie at the heart of supersymmetric quantum mechanics [10-14]. They are particularly employed for the construction of new solvable and quasiexactly solvable quantum-mechanical systems. The Darboux transformations play a fundamental role in the investigation of nonlinear equations in partial derivatives and partial difference equations, where they allow one to relate different integrable systems and provide an effective method for the construction of nontrivial solutions for them [5,15]. Periodic Darboux chains generate finite-gap systems [16] in an alternative way to the original algebro-geometric approach $[17,18]$. Such chains also generate the quantum harmonic oscillator (QHO) system and Painlevé equations [16,19,20], which are intimately related with isomonodromic deformations of linear systems and integrability properties of nonlinear systems in partial derivatives. Recently, the isomonodromic deformations [21,22] and Darboux transformations played a key role in the discovery and investigation of the properties of the new class of exceptional orthogonal polynomials [4,23-33]. One such family corresponds to exceptional Hermite polynomials, which can be

\footnotetext{
jifc@unizar.es

mikhail.plyushchay@usach.cl
}

obtained by applying Darboux and Darboux-Crum transformations to the QHO system. The quantum-mechanical systems appearing in such a way are described by certain rational extensions of the harmonic potential.

The simplest rationally extended quantum harmonic oscillator (REQHO) $[23,24]$ can be obtained from the QHO system by applying to it a Darboux transformation generated by the "Wick-rotated" second excitation of the ground state. The resulting system is characterized by an infinite tower of equidistant bound states which are separated from the ground state by a triple energy gap. As a consequence, the general solution of the evolution problem for REQHO, like for the quantum harmonic and isotonic oscillators, is periodic in time with a constant (not depending on energy) period $[23,34,35]$. For the discussion of different aspects of this quantum mechanical system, see Refs. [4,23-25,31-33].

It is known that the spectrum of the $\mathrm{QHO}$ carries an infinite-dimensional irreducible representation of the bosonized $\mathfrak{o} \mathfrak{a p}(1 \mid 2)$ superalgebra which can be generated by means of the creation and annihilation operators identified as fermionic generators $[36,37] .{ }^{1}$ In this context there appears a rather natural question: what are the ladder operators in rationally extended quantum harmonic oscillator systems and what spectrum-generating algebras do they produce? In this paper we answer these questions for the simplest case of the REQHO system by employing the properties of the Darboux transformations. A special role in the construction we obtain belongs to generalized Jordan states.

\footnotetext{
${ }^{1}$ For some recent investigations on superconformal quantummechanical symmetry and its applications, see Refs. [38-42].
} 
The paper is organized as follows. In the next section we review general properties of the Darboux transformations and related Jordan states. In Sec. III we discuss discrete flows generated by the ladder operators in the QHO system and recall the bosonized superconformal $\mathfrak{o} \mathfrak{s} \mathfrak{p}(1 \mid 2)$ structure appearing in it in the form of the spectrum generating superalgebra. In Sec. IV we generate the simplest REQHO by applying the Darboux transformation to the QHO. Then we construct ladder operators for REQHO by a Darboux dressing of the creation and annihilation operators of the QHO system, consider discrete flows, and discuss a polynomially deformed bosonized $\mathfrak{o} \mathfrak{a p}(1 \mid 2)$ structure in the REQHO that reflects in a coherent way peculiarities of its spectrum. Section V is devoted to the conclusion and outlook. In the Appendix we describe the action of the ladder operators on nonphysical eigenstates of the quantum harmonic oscillator which are closely related to its physical spectrum, and present the construction of the net of associated Jordan and generalized Jordan states.

\section{DARBOUX TRANSFORMATIONS AND JORDAN STATES}

Let $\psi_{*}(x)$ be a solution of the stationary Schrödinger equation $H \psi_{*}=E_{*} \psi_{*}$. For the moment we consider this equation formally as an abstract second-order differential equation in which $H=-\frac{d^{2}}{d x^{2}}+V(x)$ is a Hamiltonian operator with a real potential $V(x)$ that is nonsingular on $\mathbb{R}$. A real constant $E_{*}$ is treated here as an eigenvalue without considering the questions of boundary conditions and normalizability for $\psi_{*}(x)$. Consequently, we do not distinguish functions $\psi(x)$ and $C \psi(x)$, where $C \in \mathbb{C}$, $C \neq 0$, and assume that modulo such a multiplicative factor $\psi(x)$ is chosen to be a real-valued function. A linearly independent solution for the same eigenvalue $E_{*}$ can be taken in the form

$$
\widetilde{\psi_{*}}(x)=\psi_{*}(x) \int^{x} \frac{d \xi}{\psi_{*}^{2}(\xi)}
$$

Due to the integration with an indefinite lower limit, the function $\widetilde{\psi_{*}}(x)$ is supposed to be defined up to an additive term proportional to $\psi_{*}(x)$. We now assume that $E_{*}$ is chosen so that the function $\psi_{*}(x)$ is nodeless, $\psi_{*}(x) \neq 0$, and introduce the first-order differential operators

$$
A_{\psi_{*}}=\psi_{*} \frac{d}{d x} \frac{1}{\psi_{*}}=\frac{d}{d x}-\mathcal{W}(x), \quad \mathcal{W}(x)=\frac{\psi_{*}^{\prime}}{\psi_{*}},
$$

and $A_{\psi_{*}}^{\dagger}=-\frac{d}{d x}-\mathcal{W}(x)$, where a prime denotes a derivative with respect to $x$. Note that because $A_{\psi_{*}}$ and $A_{\psi_{*}}^{\dagger}$ are first-order differential operators, their kernels are one dimensional,

$$
\operatorname{ker} A_{\psi_{*}}=\psi_{*}, \quad \operatorname{ker} A_{\psi_{*}}^{\dagger}=\frac{1}{\psi_{*}} .
$$

These operators provide a factorization of the Hamiltonian shifted for the constant $E_{*}, H-E_{*}=A_{\psi_{*}}^{\dagger} A_{\psi_{*}}$. The potential $V(x)$ and superpotential $\mathcal{W}(x)$ are connected by the relation $V(x)-E_{*}=\mathcal{W}^{2}+\mathcal{W}^{\prime}$. The product with the permuted first-order operators, $A_{\psi_{*}} A_{\psi_{*}}^{\dagger}=\breve{H}-E_{*}$, defines the associated partner system described by the Hamiltonian $\breve{H}=-\frac{d^{2}}{d x^{2}}+\breve{V}(x)$ with $\breve{V}(x)-E_{*}=\mathcal{W}^{2}-\mathcal{W}^{\prime}$. From the alternative factorization relations it follows immediately that the first-order operators $A_{\psi_{*}}$ and $A_{\psi_{*}}^{\dagger}$ intertwine the quantum Hamiltonians $H$ and $\breve{H}$,

$$
A_{\psi_{*}} H=\breve{H} A_{\psi_{*}}, \quad A_{\psi_{*}}^{\dagger} \breve{H}=H A_{\psi_{*}}^{\dagger} .
$$

If $\psi_{E}$ is a physical (normalizable) or nonphysical (non-normalizable) solution of the Schrödinger equation $H \psi_{E}=E \psi_{E}$ for some eigenvalue $E \neq E_{*}$, then as a consequence of Eq. (2.4), $A_{\psi_{*}} \psi_{E}$ will be an eigenstate of $\breve{H}$ of the same (physical or nonphysical) nature for the same eigenvalue $E$ : $\breve{H}\left(A_{\psi_{*}} \psi_{E}\right)=E\left(A_{\psi_{*}} \psi_{E}\right)$. In particular, for the linearly independent solution $\widetilde{\psi_{E}}$ constructed from $\psi_{E}$ according to the rule (2.1), $\widetilde{\psi_{E}}=\psi_{E}(x) \int^{x} d \xi / \psi_{E}^{2}(\xi)$, we have $\breve{H}\left(A_{\psi_{*}} \widetilde{\psi_{E}}\right)=E\left(A_{\psi_{*}} \widetilde{\psi_{E}}\right)$. On the other hand, for $E=E_{*}$ and $\psi_{E}=\widetilde{\psi_{*}}$ we find that

$$
A_{\psi_{*}} \widetilde{\psi_{*}}=\frac{1}{\psi_{*}} .
$$

The function $\frac{1}{\psi_{*}}$ is the kernel of the operator $A_{\psi_{*}}^{\dagger}$, and therefore it is an eigenstate of $\breve{H},\left(\breve{H}-E_{*}\right) \frac{1}{\psi_{*}}=0$. Analogously, if $\breve{\psi}_{E}$ is an eigenfunction of $\breve{H}$ of eigenvalue $E \neq E_{*}$, then $A_{\psi_{*}}^{\dagger} \breve{\psi}_{E}$ is an eigenstate of $H$ of the same eigenvalue, $H\left(A_{\psi_{*}}^{\dagger} \breve{\psi}_{E}\right)=E\left(A_{\psi_{*}}^{\dagger} \breve{\psi}_{E}\right)$. For $E=E_{*}$ the application of $A_{\psi_{*}}^{\dagger}$ to an eigenfunction of $\breve{H}$ that is linearly independent from $\frac{1}{\psi_{*}}$ maps it into the kernel of $A_{\psi_{*}}$,

$$
A_{\psi_{*}}^{\dagger} \widetilde{\left(\frac{1}{\psi_{*}}\right)}=\psi_{*},
$$

which is the eigenstate of $H$.

The described structure of the Darboux transformations reveals an essential difference between the cases $E \neq E_{*}$ and $E=E_{*}$. The action of the Darboux transformation generators on the eigenstates with $E \neq E_{*}$ is of the twocyclic nature in the following sense: if $\psi$ is such that $H \psi=E \psi$, then $A_{\psi *}$ maps this state into an eigenstate of $\breve{H}$, $A_{\psi_{*}} \psi=\breve{\psi}, \breve{H} \breve{\psi}=E \breve{\psi}$, while the application of $A_{\psi_{*}}^{\dagger}$ to $\breve{\psi}$ reproduces (up to a multiplicative factor) the initial state $\psi$. At the same time, for $E=E_{*}$ we have $A_{\psi_{*}} \widetilde{\psi_{*}}=\frac{1}{\psi_{*}}$, 

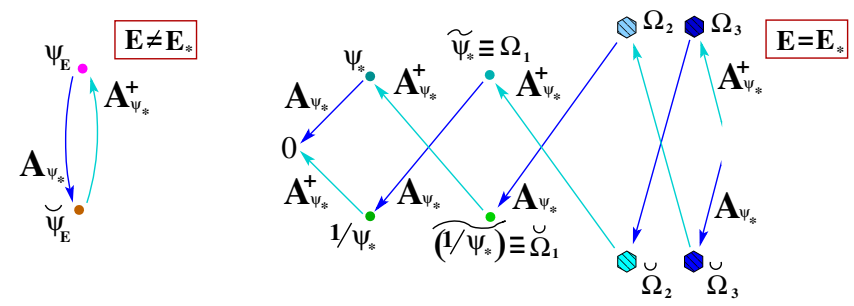

FIG. 1. Action of the Darboux transformation generators.

$A_{\psi_{*}}^{\dagger} \frac{1}{\psi_{*}}=0$ and $A_{\psi_{*}}^{\dagger} \widetilde{\left(\frac{1}{\psi_{*}}\right)}=\psi_{*}, A_{\psi_{*}} \psi_{*}=0$, and no analogous cyclic structure appears. In this case the functions

$$
\begin{aligned}
& \Omega_{2}(x)=\psi_{*}(x) \int^{x} \frac{1}{\psi_{*}(\xi)} \widetilde{\left(\frac{1}{\psi_{*}}\right)}(\xi) d \xi, \\
& \breve{\Omega}_{2}(x)=\frac{1}{\psi_{*}(x)} \int^{x} \psi_{*}(\xi) \widetilde{\psi_{*}}(\xi) d \xi
\end{aligned}
$$

are the preimages of $\widetilde{\left(\frac{1}{\psi_{*}}\right)}$ and $\widetilde{\psi_{*}}$,

$$
A_{\psi_{*}} \Omega_{2}=\widetilde{\left(\frac{1}{\psi_{*}}\right)} \equiv \breve{\Omega}_{1}, \quad A_{\psi_{*}}^{\dagger} \breve{\Omega}_{2}=\widetilde{\psi_{*}} \equiv \Omega_{1} .
$$

Similarly to the eigenstates of the form (2.1), the functions $\Omega_{2}(x)$ and $\breve{\Omega}_{2}(x)$ are defined modulo additive terms $\psi_{*}(x)$ and $\frac{1}{\psi_{*}(x)}$, respectively. The wave functions (2.7) are not, however, formal eigenfunctions of $H$ and $\breve{H}$, but as a consequence of Eq. (2.8) they obey the relations $A_{\psi_{*}} A_{\psi_{*}}^{\dagger} A_{\psi_{*}} \Omega_{2}=0$ and $A_{\psi_{*}}^{\dagger} A_{\psi_{*}} A_{\psi_{*}}^{\dagger} \breve{\Omega}_{2}=0$. Therefore,

$$
\left(H-E_{*}\right)^{2} \Omega_{2}=0, \quad\left(\breve{H}-E_{*}\right)^{2} \breve{\Omega}_{2}=0,
$$

and we conclude that $\Omega_{2}$ and $\breve{\Omega}_{2}$ are generalized eigenstates of $H$ and $\breve{H}$ of rank 2 corresponding to the same eigenvalue $E=E_{*}$. Having in mind a generalization of relations of the form (2.9) which appear in the following [in particular, see Eq. (4.16) below], we refer to $\Omega_{2}$ and $\breve{\Omega}_{2}$ as Jordan states of order 2 [43,44]. The states $(2.7)$ can be generalized further by defining

$$
\begin{aligned}
& \Omega_{n}(x)=\psi_{*}(x) \int^{x} \frac{1}{\psi_{*}(\xi)} \breve{\Omega}_{n-1}(\xi) d \xi, \\
& \breve{\Omega}_{n}(x)=\frac{1}{\psi_{*}(x)} \int^{x} \psi_{*}(\xi) \Omega_{n-1}(\xi) d \xi,
\end{aligned}
$$

where $n=2,3, \ldots$. These states obey the relations $A_{\psi_{*}} \Omega_{n}=\breve{\Omega}_{n-1}, \quad A_{\psi_{*}}^{\dagger} \breve{\Omega}_{n}=\Omega_{n-1}$. Consequently we find that $\Omega_{n}$ and $\breve{\Omega}_{n}$ are annihilated by differential operators of order $n+1$ constructed in terms of $A_{\psi_{*}}$ and $A_{\psi_{*}}^{\dagger}$. Namely, for even $n=2 k$ we have $A_{\psi_{*}}\left(A_{\psi_{*}}^{\dagger} A_{\psi_{*}}\right)^{k} \Omega_{2 k}=0$, $A_{\psi_{*}}^{\dagger}\left(A_{\psi_{*}} A_{\psi_{*}}^{\dagger}\right)^{k} \breve{\Omega}_{2 k}=0$, while for odd $n=2 k-1$ we obtain
$\left(A_{\psi_{*}}^{\dagger} A_{\psi_{*}}\right)^{k} \Omega_{2 k-1}=0$ and $\left(A_{\psi_{*}} A_{\psi_{*}}^{\dagger}\right)^{k} \breve{\Omega}_{2 k-1}=0, k=1, \ldots$ In both cases of the even and odd values of $n$ we have $\left(H-E_{*}\right)^{n+1} \Omega_{2 n}=0,\left(\breve{H}-E_{*}\right)^{n+1} \breve{\Omega}_{2 n}=0, n=1,2, \ldots$, and $\quad\left(H-E_{*}\right)^{n+1} \Omega_{2 n+1}=0, \quad\left(\breve{H}-E_{*}\right)^{n+1} \breve{\Omega}_{2 n+1}=0$, $n=0,1, \ldots$ Thus, $\Omega_{k}$ and $\breve{\Omega}_{k}$ with $k=2 n, 2 n+1$ are Jordan states of order $n+1$. The described properties are illustrated in Fig. 1.

\section{DISCRETE FLOWS IN THE HARMONIC OSCILLATOR SYSTEM AND THE $\mathfrak{o} \mathfrak{g} \mathfrak{p}(1 \mid 2)$}

Let us consider now the QHO system with $V(x)=x^{2}$. Its physical bound eigenstates with eigenvalues $E_{n}=2 n+1$ are described by normalizable wave functions $\psi_{n}(x)=$ $H_{n}(x) e^{-x^{2} / 2}, n=0,1, \ldots$, where $H_{n}(x)$ are the Hermite polynomials. The change $x \rightarrow i x$ transforms the Hamiltonian of the QHO

$$
H=-\frac{d^{2}}{d x^{2}}+x^{2}
$$

into $-H$, from which it follows that the wave functions $\psi_{n}^{-}(x)=\mathcal{H}_{n}(x) e^{x^{2} / 2}$ with $\mathcal{H}_{n}(x) \equiv H_{n}(i x)$ correspond to nonphysical (i.e., non-normalizable) eigenstates of $H$ with eigenvalues $E_{n}^{-}=-(2 n+1)$. The corresponding functions $\widetilde{\psi_{n}}$ and $\widetilde{\psi_{n}^{-}}$are nonphysical (non-normalizable) eigenfunctions of $H$ with eigenvalues $E_{n}=2 n+1$ and $E_{n}=$ $-(2 n+1), n=0,1, \ldots$, respectively. They will play an important role in the structure and properties of the REQHO system [4,23-25,31-33].

The well-known peculiarity of the QHO system in the context of the Darboux transformations is that the choice of $E_{*}=1, \psi_{*}=\psi_{0}=e^{-x^{2} / 2}$ gives $\mathcal{W}=-x$, and with the factorizing operators $A_{\psi_{*}}$ and $A_{\psi_{*}}^{\dagger}$ we obtain the usual (up to a multiplicative factor $\sqrt{2}$ ) creation and annihilation operators,

$a^{-}=\frac{d}{d x}+x, \quad a^{+}=\left(a^{-}\right)^{\dagger}=-\frac{d}{d x}+x, \quad\left[a^{-}, a^{+}\right]=2$.

In this case, if $N=a^{+} a^{-}$denotes the number operator for the QHO [with the spectrum $2 n, n=0,1, \ldots$, corresponding to a normalization chosen in Eq. (3.2)], we have

$$
\left[N, a^{ \pm}\right]= \pm 2 a^{ \pm}, \quad H=N+1 .
$$

As a result, the Darboux-partner system for the QHO turns out to be $\breve{H}=H+2$, which is the same quantum harmonic oscillator but just with the spectrum of physical states shifted by +2 . Since $\psi_{0}^{-}=1 / \psi_{0}$, another choice $E_{*}=-1$, $\psi_{*}=\psi_{0}^{-}$changes the role of the Darboux-generating operators, $A_{\psi_{*}}=a^{+}, A_{\psi_{*}}^{\dagger}=a^{-}$, and the partner system $\breve{H}=H-2$ in this second case is again the quantum 


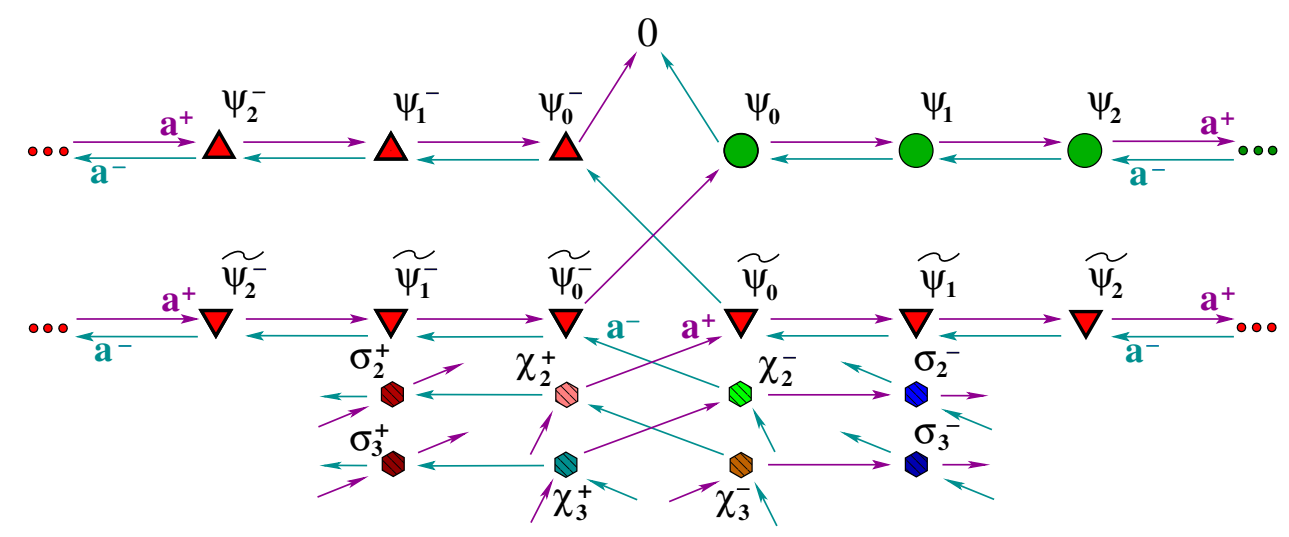

FIG. 2. Discrete flows of the ladder operators of the QHO. The operator $a^{-}$acts left and up, and $a^{+}$acts right and up.

harmonic oscillator but with the physical spectrum shifted by -2 . The action of the ladder operators on physical and associated nonphysical eigenstates of the QHO and on the related Jordan and generalized Jordan states is described in the Appendix. The corresponding discrete flows generated by $a^{-}$and $a^{+}$are depicted in Fig. 2.

In general, because of the two-cyclic structure associated with the Darboux transformations, there appears a supersymmetry in the extended system composed from $H$ and $\breve{H}$. Since for the QHO with its equidistant spectrum the partner generated by the Darboux transformation based on the eigenfunction $\psi_{*}=\psi_{0}$ ( or on $\psi_{*}=\psi_{0}^{-}=1 / \psi_{0}$ ) is the same system but just with the spectrum shifted exactly by one energy step $\Delta E=+2$ (or, $\Delta E=-2$ ), the Darboux transformation generators responsible for the supersymmetric structure transmute into the ladder operators for the single harmonic oscillator system. In addition, instead of the usual quantum-mechanical supersymmetry of the composed system, the single quantum harmonic oscillator itself is characterized by the bosonized superconformal $\mathfrak{o} \mathfrak{g} \mathfrak{p}(1 \mid 2)$ structure. The $\mathfrak{o} \mathfrak{g} \mathfrak{p}(1 \mid 2)$ Lie superalgebra is generated here by the set of operators

$\mathcal{L}_{ \pm}=\frac{1}{4} a^{ \pm}, \quad J_{0}=\frac{1}{8}\left\{a^{+}, a^{-}\right\}=\frac{1}{4} H, \quad J_{ \pm}=\frac{1}{4}\left(a^{ \pm}\right)^{2}$,

with nontrivial (anti)commutation relations

$$
\left\{\mathcal{L}_{+}, \mathcal{L}_{-}\right\}=\frac{1}{2} J_{0}, \quad\left\{\mathcal{L}_{+}, \mathcal{L}_{+}\right\}=\frac{1}{2} J_{+}, \quad\left\{\mathcal{L}_{-}, \mathcal{L}_{-}\right\}=\frac{1}{2} J_{-}
$$

$\left[J_{0}, \mathcal{L}_{ \pm}\right]= \pm \frac{1}{2} \mathcal{L}_{ \pm}, \quad\left[J_{+}, \mathcal{L}_{-}\right]=-\mathcal{L}_{+}, \quad\left[J_{-}, \mathcal{L}_{+}\right]=\mathcal{L}_{-}$,

$$
\left[J_{0}, J_{ \pm}\right]= \pm J_{ \pm}, \quad\left[J_{+}, J_{-}\right]=-2 J_{0} .
$$

For this superalgebra the reflection operator $\mathcal{R}=$ $(-1)^{N / 2}=e^{i \pi N / 2}$ plays the role of a $\mathbb{Z}_{2}$-grading operator, i.e.,

$\mathcal{R}^{2}=1, \quad\left\{\mathcal{R}, \mathcal{L}_{ \pm}\right\}=0, \quad\left[\mathcal{R}, J_{0}\right]=\left[\mathcal{R}, J_{ \pm}\right]=0$.

The operator

$\mathcal{C}_{\mathfrak{o} \mathfrak{g} \mathfrak{p}(1 \mid 2)}=-J_{0}^{2}+\frac{1}{2}\left(J_{+} J_{-}+J_{-} J_{+}\right)+2\left(\mathcal{L}_{+} \mathcal{L}_{-}-\mathcal{L}_{-} \mathcal{L}_{+}\right)$

is the quadratic Casimir of the $\mathfrak{o} \mathfrak{s} \mathfrak{p}(1 \mid 2)$.

This corresponds to the well-known spectrum-generating superalgebra of the QHO [36,37], on the physical eigenstates $\psi_{n}(x)$ of which the infinite-dimensional irreducible representation of the $\mathfrak{o} \mathfrak{g} \mathfrak{p}(1 \mid 2)$ with $\mathcal{C}_{\mathfrak{o} \mathfrak{g} \mathfrak{p}(1 \mid 2)}=-\frac{1}{16}$ is realized. The generators of the $\mathfrak{g} \mathfrak{v}(2,1)$ Lie subalgebra $(3.7)$ act irreducibly on the eigensubspaces of $\mathcal{R}$ spanned by the states $\psi_{n}(x)$ with even $\left(n=2 n_{+}\right)$and odd $\left(n=2 n_{-}+1\right.$, $n_{ \pm}=0,1, \ldots$ ) values of $n$, where the operator $J_{0}$ takes eigenvalues $n_{+}+\frac{1}{4}$ and $n_{-}+\frac{3}{4}$, respectively. On both these subspaces the $\mathfrak{g} \mathfrak{p}(2,1)$ Casimir operator $\mathcal{C}_{\mathfrak{g} \mathfrak{p}(2,1)}=-J_{0}^{2}+$ $\frac{1}{2}\left(J_{+} J_{-}+J_{-} J_{+}\right)$takes the same value $\mathcal{C}_{\mathfrak{g} \mathfrak{o}(2,1)}=\frac{3}{16}$.

To conclude this section we note that a structure with a hidden bosonized supersymmetry $[45,46]$ also appears in periodic finite-gap and reflectionless quantum-mechanical systems [47]. There, however, hidden supersymmetry has a different origin associated with the presence of a nontrivial Lax-Novikov integral in the quantum-mechanical systems related to finite-gap and soliton solutions of the Kortewegde Vries equation.

\section{DISCRETE FLOWS IN THE REQHO AND DEFORMED SUPERCONFORMAL $\mathfrak{o} \mathfrak{g p}(\mathbf{1} \mid 2)$ STRUCTURE}

Now we take a nonphysical eigenstate $\psi_{2}^{-}(x)=\left(2 x^{2}+\right.$ 1) $e^{x^{2} / 2}$ of the harmonic oscillator as the function $\psi_{*}$ to 
generate the Darboux transformation. This is a nodeless function, and the associated Darboux-transformed system will be given by a potential that is nonsingular on $\mathbb{R}$. For the sake of simplicity we denote by $A^{-}$the corresponding first-order operator (2.2), in which the superpotential

$\mathcal{W}(x)=\frac{d}{d x}\left(\ln \psi_{2}^{-}\right)=x+\frac{4 x}{2 x^{2}+1}=x+\frac{1}{x+\frac{i}{\sqrt{2}}}+\frac{1}{x-\frac{i}{\sqrt{2}}}$

has simple poles at $\infty$ and $\pm \frac{i}{\sqrt{2}}$. By construction, $A^{-} \psi_{2}^{-}=0$ and $A^{+}\left(\frac{1}{\psi_{2}^{-}}\right)=0$, where $A^{+}=\left(A^{-}\right)^{\dagger}$. A simple computation gives

$$
\begin{array}{r}
A^{+} A^{-}=-\frac{d^{2}}{d x^{2}}+x^{2}+5=N+6 \equiv H_{O}, \\
A^{-} A^{+}=-\frac{d^{2}}{d x^{2}}+x^{2}+3+8 \frac{2 x^{2}-1}{\left(2 x^{2}+1\right)^{2}} \equiv \breve{H}_{O},
\end{array}
$$

where $N$ is the number operator for the QHO, $N=a^{+} a^{-}$. Here $H_{O}$ represents the QHO Hamiltonian shifted by an additive constant 5. The Hamiltonian operator $\breve{H}_{O}$ describes the REQHO system with the physical bound states $\Psi_{0}=\frac{1}{\psi_{2}^{-}}=A^{-} \widetilde{\psi_{2}^{-}}$and $\Psi_{n+1}=A^{-} \psi_{n}$ of energies $E_{0}=0$ and $E_{n+1}=6+2 n, n=0,1, \ldots$, constructed from the corresponding $\mathrm{QHO}$ states.

Let us introduce the third-order differential operators

$$
\mathcal{A}^{-}=A^{-} a^{-} A^{+}, \quad \mathcal{A}^{+}=\left(\mathcal{A}^{-}\right)^{\dagger}=A^{-} a^{+} A^{+} .
$$

These are the Darboux-dressed ladder operators of the QHO. The operator $A^{+}$maps a physical or nonphysical eigenstate of $\breve{H}_{O}$ into an eigenstate (of the same nature) of the QHO, to which $a^{-}$or $a^{+}$is then applied, and the eigenstate of $H_{O}$ obtained in this way is mapped by $A^{-}$into another eigenstate of $\breve{H}_{O}$. The operators (4.4) satisfy the following commutation relations with the REQHO Hamiltonian:

$$
\left[\breve{H}, \mathcal{A}^{ \pm}\right]= \pm 2 \mathcal{A}^{ \pm}
$$

for which from now on we use a simplified notation $\breve{H}$. To find Eq. (4.5) we used the intertwining relations $A^{+} \breve{H}=H_{O} A^{+}, A^{-} H_{O}=\breve{H} A^{-}$, as well as Eq. (3.3). Equation (4.5) is generalized further for

$$
\left[\breve{H}, \mathcal{A}^{ \pm n}\right]= \pm 2 n \mathcal{A}^{ \pm n}, \quad n=1,2, \ldots,
$$

and $f(\breve{H}) \mathcal{A}^{ \pm}=\mathcal{A}^{ \pm} f(\breve{H} \pm 2)$ for an arbitrary polynomial function $f(\breve{H})$.

The operators $\mathcal{A}^{+}$and $\mathcal{A}^{-}$transform eigenstates of $\breve{H}$ (which are not from their kernels) into eigenstates of $\breve{H}$ with the energy values increased and decreased by 2 . In this aspect they act analogously to the ladder operators $a^{+}$and $a^{-}$in the QHO system. There are, however, essential differences. These third-order differential operators satisfy the relations

$$
\begin{aligned}
& \mathcal{A}^{+} \mathcal{A}^{-}=\breve{H}(\breve{H}-2)(\breve{H}-6) \equiv \Phi(\breve{H}), \\
& \mathcal{A}^{-} \mathcal{A}^{+}=\breve{H}(\breve{H}+2)(\breve{H}-4)=\Phi(\breve{H}+2),
\end{aligned}
$$

which follow from Eqs. (4.2), (4.3), and (3.3) and the intertwining properties of $A^{ \pm}$, and include the degree-three polynomial $\Phi(\lambda)=\lambda(\lambda-2)(\lambda-6)$. From Eqs. (4.7) and (4.6) we also obtain the following relations which will be used below:

$$
\begin{aligned}
\mathcal{A}^{+2} \mathcal{A}^{-}= & \Phi(\breve{H}-2) \mathcal{A}^{+}, \quad \mathcal{A}^{-} \mathcal{A}^{+2}=\Phi(\breve{H}+2) \mathcal{A}^{+}, \\
\mathcal{A}^{-2} \mathcal{A}^{+}= & \Phi(\breve{H}+4) \mathcal{A}^{-}, \quad \mathcal{A}^{+} \mathcal{A}^{-2}=\Phi(\breve{H}) \mathcal{A}^{-}, \\
& \mathcal{A}^{+2} \mathcal{A}^{-2}=\Phi(\breve{H}) \Phi(\breve{H}-2), \\
& \mathcal{A}^{-2} \mathcal{A}^{+2}=\Phi(\breve{H}+2) \Phi(\breve{H}+4) .
\end{aligned}
$$

Both third-order polynomials $\Phi(\breve{H})$ and $\Phi(\breve{H}+2)$ in Eq. (4.7) include a factor $\breve{H}$. This reflects the essential peculiarity of the REQHO system: its ground state $\Psi_{0}$ of zero energy is annihilated by both operators $\mathcal{A}^{-}$and $\mathcal{A}^{+}$,

$$
\mathcal{A}^{-} \Psi_{0}=\mathcal{A}^{+} \Psi_{0}=0,
$$

because $\Psi_{0}=\frac{1}{\psi_{2}^{-}}$is the kernel of $A^{+}$.

Now we consider other properties of the lowering ladder operator $\mathcal{A}^{-}$. It also annihilates the first excited physical state $\Psi_{1}(x)=A^{-} \psi_{0}(x), \mathcal{A}^{-} \Psi_{1}=0$ due to the sequential action of the operators $A^{+}$and $a^{-}$. Moreover, it annihilates a nonphysical eigenstate $A^{-} \psi_{1}^{-}$of $\breve{H}$ by means of transforming it by the second-order operator $a^{-} A^{+}$into the kernel of $A^{-}$. As the kernel of the third-order differential operator $\mathcal{A}^{-}$is three dimensional, it is spanned by the three eigenstates of $\breve{H}$,

$$
\operatorname{ker} \mathcal{A}^{-}=\operatorname{span}\left\{\Psi_{0}, A^{-} \psi_{1}^{-}, \Psi_{1}\right\},
$$

whose eigenvalues $E=0,2,6$ correspond to zeros of the third-degree polynomial $\Phi(\breve{H})$ in the first equality in Eq. (4.7). The operator $\mathcal{A}^{-}$acts as a lowering ladder operator, and it is also interesting to look for kernels of powers $\left(\mathcal{A}^{-}\right)^{n}$ with $n=2,3, \ldots$ A priori, it is clear thatdue to the presence of another physical state in the kernel of $\mathcal{A}^{-}$(which is the first exited state $\Psi_{1}$ in the spectrum of $\breve{H}$ ) and of the nonphysical state $A^{-} \psi_{1}^{-}$with eigenvalue $E=2$ located between the energies $E=0$ and $E=6$ of the physical zero modes of $\mathcal{A}^{-}$- some additional peculiarities have to appear in comparison with the case of the QHO. 


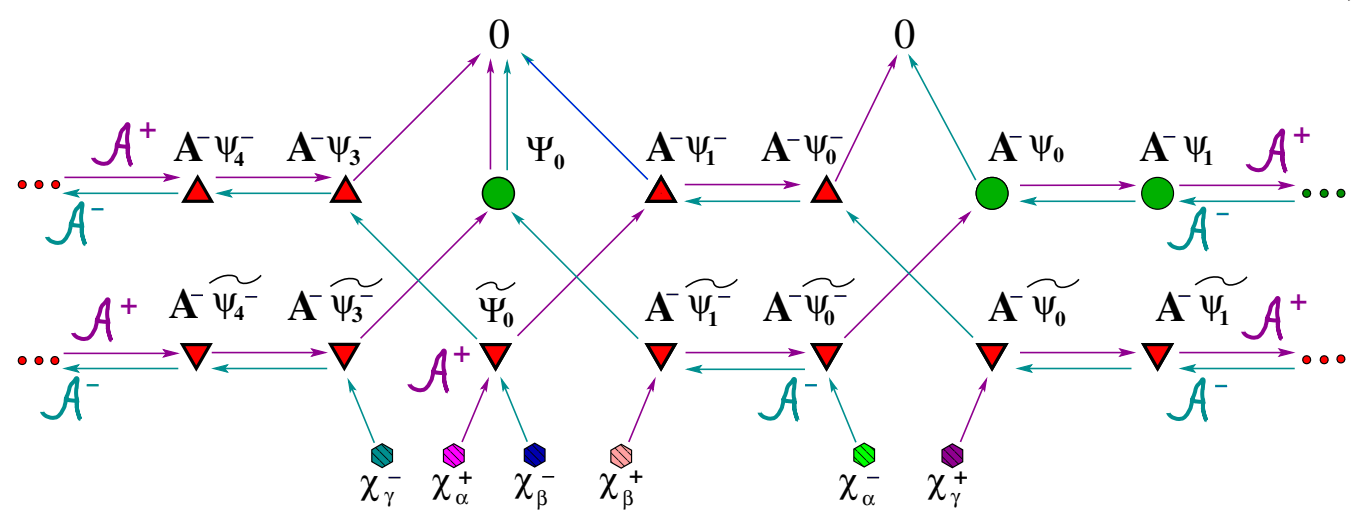

FIG. 3. Discrete flows of the ladder operators of the REQHO. The operator $\mathcal{A}^{-}$acts left and up, and $\mathcal{A}^{+}$acts right and up.

Note first that Eq. (4.6) implies that $\operatorname{ker}\left(\mathcal{A}^{-}\right)^{2}$ must be invariant under the action of $\breve{H}$. On the other hand, we remark that $\operatorname{ker} \mathcal{A}^{-} \subset \operatorname{ker}\left(\mathcal{A}^{-}\right)^{2}$. Moreover, $\psi \in \operatorname{ker}\left(\mathcal{A}^{-}\right)^{2}$ if and only if $\mathcal{A}^{-}(\psi) \in \operatorname{ker} \mathcal{A}^{-}$, and therefore $\operatorname{ker}\left(\mathcal{A}^{-}\right)^{2}$ is generated by $\operatorname{ker} \mathcal{A}^{-}$and the preimages under $\mathcal{A}^{-}$of $\Psi_{0}$, $A^{-} \psi_{1}^{-}$and $\Psi_{1}$, i.e., one finds that

$$
\operatorname{ker}\left(\mathcal{A}^{-}\right)^{2}=\operatorname{span}\left\{\operatorname{ker} \mathcal{A}^{-}, A^{-} \widetilde{\psi_{1}^{-}}, A^{-} \psi_{0}^{-}, \Psi_{2}\right\}
$$

Here $\Psi_{2}=A^{-} \psi_{1}$ is a physical eigenstate at the next energy level $E=8$, and two other states $A^{-} \widetilde{\psi_{1}^{-}}$and $A^{-} \psi_{0}^{-}$are nonphysical eigenstates of $\breve{H}$ of energies $E=2$ and $E=4$. Under the action of $\mathcal{A}^{-}$the states $A^{-} \widetilde{\psi_{1}^{-}}, A^{-} \psi_{0}^{-}$, and $\Psi_{2}$ are transformed into the states $\Psi_{0}, A^{-} \psi_{1}^{-}$, and $\Psi_{1}$ from the kernel (4.12). One can proceed in this way and identify the action of the decreasing operator on all the physical eigenstates of the system and on the associated nonphysical eigenstates of the special form $\widetilde{\Psi_{0}}, A^{-} \widetilde{\psi_{n}}, n=0,1, \ldots$, and $A^{-} \psi_{n}^{-}, A^{-} \widetilde{\psi_{n}^{-}}, n=0,1,3,4,5, \ldots$ This action is depicted in Fig. 3. The figure also shows that the preimages of all the indicated physical and nonphysical eigenstates of $\breve{H}$ with eigenvalues $E_{n}=2 n, n \in \mathbb{Z}$, are contained in the same set of eigenstates with the exception of the three nonphysical states $A^{-} \widetilde{\psi_{0}^{-}}, \widetilde{\Psi_{0}}=\widetilde{\left(\frac{1}{\psi_{2}^{-}}\right)}$, and $A^{-} \widetilde{\psi_{3}^{-}}$of the eigenvalues $E=4, E=0$, and $E=-2$, respectively. These eigenvalues coincide with the set of zeros of the polynomial $\Phi(\breve{H}+2)$ that appears in the second relation in Eq. (4.7). The preimages of the indicated states are the states $\chi_{a}^{-}, a=\alpha, \beta, \gamma$, which have the structure

$\chi_{a}^{-}(x)=\frac{1}{\psi_{2}^{-}(x)} \int^{x} \psi_{0}(\xi) \psi_{2}^{-}(\xi)\left(\int^{\xi} \psi_{0}^{-}(\eta) \rho_{a}^{-}(\eta) d \eta\right) d \xi$.

Here $\rho_{\alpha}^{-}=\widetilde{\psi_{0}^{-}}, \rho_{\beta}^{-}=\Omega_{\psi_{2}^{-}}, \rho_{\gamma}^{-}=\widetilde{\psi_{3}^{-}}$, and $\mathcal{A}^{-} \chi_{\alpha}^{-}=A^{-} \widetilde{\psi_{0}^{-}}$, $\mathcal{A}^{-} \chi_{\beta}^{-}=\widetilde{\Psi_{0}}, \mathcal{A}^{-} \chi_{\gamma}^{-}=A^{-} \widetilde{\psi_{3}^{-}}$. The states $\chi_{a}^{-}$are not eigenstates of $\breve{H}$ but satisfy the relations
$\breve{H}(\breve{H}-6) \chi_{\alpha}^{-}=A^{-} \psi_{0}, \quad \breve{H}(\breve{H}-2)(\breve{H}-6) \chi_{\beta}^{-}=A^{-} \psi_{1}^{-}$,

$\breve{H}(\breve{H}-6) \chi_{\gamma}^{-}=\Psi_{0}$.

This implies that the following polynomials in the Hamiltonian $\breve{H}$ annihilate the states $\chi_{a}^{-}$:

$\breve{H}(\breve{H}-6)^{2} \chi_{\alpha}^{-}=0, \quad \breve{H}(\breve{H}-2)^{2}(\breve{H}-6) \chi_{\beta}^{-}=0$,

$\breve{H}^{2}(\breve{H}-6) \chi_{\gamma}^{-}=0$.

In correspondence with Eq. (4.16), which generalizes the relations (2.9), we call the states $\chi_{a}^{-}$the generalized Jordan states of the REQHO since they are destroyed by the polynomials in $\breve{H}$ with different roots. Note also that

$$
\left(\mathcal{A}^{-}\right)^{3} \chi_{\alpha}^{-}=\Psi_{0} \Rightarrow \chi_{\alpha}^{-} \in \operatorname{ker}\left(\mathcal{A}^{-}\right)^{4},
$$

whereas $\chi_{\beta}^{-}$and $\chi_{\gamma}^{-}$are not annihilated by any degree of the ladder operator $\mathcal{A}^{-}$and in this aspect they are similar to the Jordan state $\chi_{2}^{-}$in the QHO system; see Eq. (A6).

The kernel of the raising ladder operator is

$$
\operatorname{ker} \mathcal{A}^{+}=\operatorname{span}\left\{A^{-} \psi_{3}^{-}, \Psi_{0}, A^{-} \psi_{0}^{-}\right\} \text {. }
$$

The action of $\mathcal{A}^{+}$is also illustrated in Fig. 3. The corresponding generalized Jordan states $\chi_{a}^{+}, a=\alpha, \beta, \gamma$, shown there are given by relations similar to Eq. (4.14),

$\chi_{a}^{+}(x)=\frac{1}{\psi_{2}^{-}(x)} \int^{x} \psi_{0}^{-}(\xi) \psi_{2}^{-}(\xi)\left(\int^{\xi} \psi_{0}(\eta) \rho_{a}^{+}(\eta) d \eta\right) d \xi$

where $\rho_{\alpha}^{+}=\Omega_{\psi_{2}^{-}}, \rho_{\beta}^{+}=\widetilde{\psi_{1}^{-}}, \rho_{\gamma}^{+}=\widetilde{\Psi_{0}}$, and $\mathcal{A}^{+} \chi_{\alpha}^{+}=\widetilde{\Psi_{0}}$, $\mathcal{A}^{+} \chi_{\beta}^{+}=A^{-} \widetilde{\psi_{1}^{-}}, \mathcal{A}^{+} \chi_{\gamma}^{+}=A^{-} \widetilde{\Psi_{0}}$. The nonphysical eigenstates $\widetilde{\Psi_{0}}, A^{-} \widetilde{\psi_{1}^{-}}$and $A^{-} \widetilde{\Psi_{0}}$ of $\breve{H}$ appearing here have eigenvalues $E=0, E=2$, and $E=6$, respectively, which correspond to zeros of the polynomial $\Phi(\breve{H})$ in Eq. (4.7). The states $\chi_{a}^{+}$satisfy the relations 


$$
\begin{aligned}
\breve{H}(\breve{H}+2)(\breve{H}-4) \chi_{\alpha}^{+} & =A^{-} \psi_{3}^{-}, \quad \breve{H}(\breve{H}-4) \chi_{\beta}^{+}=\Psi_{0}, \\
\breve{H}(\breve{H}-4) \chi_{\gamma}^{+} & =A^{-} \psi_{0}^{-} .
\end{aligned}
$$

As a consequence,

$$
\begin{aligned}
\breve{H}(\breve{H}+2)^{2}(\breve{H}-4) \chi_{\alpha}^{+}=0, \quad \breve{H}^{2}(\breve{H}-4) \chi_{\beta}^{+}=0, \\
\breve{H}(\breve{H}-4)^{2} \chi_{\gamma}^{+}=0 .
\end{aligned}
$$

Note that we have here

$$
\left(\mathcal{A}^{+}\right)^{3} \chi_{\alpha}^{+}=A^{-} \psi_{0}^{-} \Rightarrow \chi_{\alpha}^{+} \in \operatorname{ker}\left(\mathcal{A}^{+}\right)^{4},
$$

cf. Eq. (4.17). The notations for the generalized Jordan states $\chi_{a}^{ \pm}$are chosen so that the ordering in the lower index $a=\alpha, \beta, \gamma$ in the wave functions $\chi_{a}^{+}$corresponds to the ordering in the energies of the associated nonphysical eigenstates $\widetilde{\Psi_{0}}, A^{-} \widetilde{\psi_{1}^{-}}$, and $A^{-} \widetilde{\Psi_{0}}$. The generalized Jordan state $\chi_{\alpha}^{-}$is characterized by the property (4.17) to be similar to the property (4.22) for $\chi_{\alpha}^{+}$. Under subsequent application of the ladder operator $\mathcal{A}^{+}$to the state $\chi_{\beta}^{+}$and of the operator $\mathcal{A}^{-}$ to $\chi_{\beta}^{-}$, these states are lifted up to the highest horizontal level shown in Fig. 3 to which physical eigenstates belong, while the generalized Jordan states $\chi_{\gamma}^{+}$and $\chi_{\gamma}^{-}$ are lifted up by analogous action of the corresponding ladder operator to the lower horizontal level where only nonphysical eigenstates of $\breve{H}$ appear. As in the case of the QHO system, one can proceed and construct iteratively the net of the related generalized Jordan states by finding the preimages and images of the six basic generalized Jordan states $\chi_{a}^{ \pm}$, and of the new states generated in such a way under the sequential action of the ladder operators $\mathcal{A}^{ \pm}$.

A complete isolation of the ground state $\Psi_{0}$ from other normalizable eigenstates $\Psi_{n}$ with $n=1,2, \ldots$, reflects here the fact that two irreducible representations of the polynomially deformed superconformal algebra $\mathfrak{o} \mathfrak{s} \mathfrak{p}(1 \mid 2)$ are realized on the physical bound states of the REQHO system. The operators $\breve{\mathcal{L}}_{ \pm}=\frac{1}{4} \mathcal{A}^{ \pm}$can be identified as the odd generators of the superalgebra, $\left\{\mathcal{R}, \breve{\mathcal{L}}_{ \pm}\right\}=0$, while $\breve{J}_{0}=\frac{1}{4} \breve{H}$ and $\breve{J}_{ \pm}=\frac{1}{4} \mathcal{A}^{ \pm 2}$ are its even generators, $\left[\mathcal{R}, \breve{J}_{0}\right]=\left[\mathcal{R}, \breve{J}_{ \pm}\right]=0$. Here, as in the case of the QHO, the operator $\mathcal{R}=(-1)^{N / 2}$ with $N=a^{+} a^{-}$is the $\mathbb{Z}_{2^{-}}$ grading operator, $\mathcal{R}^{2}=1$. The nontrivial commutation and anticommutation relations of the deformed $\mathfrak{o} \mathfrak{s} \mathfrak{p}(1 \mid 2)$ superalgebra of the REQHO can be found with the help of Eqs. (4.5)-(4.10). They can be presented in the form

$$
\begin{aligned}
\left\{\breve{\mathcal{L}}_{+}, \breve{\mathcal{L}}_{-}\right\} & =\frac{1}{2} \mathcal{C}_{\mathcal{L L}}\left(\breve{J}_{0}\right) \breve{J}_{0}, \quad\left\{\breve{\mathcal{L}}_{+}, \breve{\mathcal{L}}_{+}\right\}=\frac{1}{2} \breve{J}_{+}, \\
\left\{\breve{\mathcal{L}}_{-}, \breve{\mathcal{L}}_{-}\right\} & =\frac{1}{2} \breve{J}_{-},
\end{aligned}
$$

$$
\begin{aligned}
{\left[\breve{J}_{0}, \breve{\mathcal{L}}_{ \pm}\right] } & = \pm \frac{1}{2} \breve{\mathcal{L}}_{ \pm}, \quad\left[\breve{J}_{+}, \breve{\mathcal{L}}_{-}\right]=-\mathcal{C}_{J \mathcal{L}}\left(\breve{J}_{0}\right) \breve{\mathcal{L}}_{+}, \\
{\left[\breve{J}_{-}, \breve{\mathcal{L}}_{+}\right] } & =\breve{\mathcal{L}}_{-} \mathcal{C}_{J \mathcal{L}}\left(\breve{J}_{0}\right), \\
{\left[\breve{J}_{0}, \breve{J}_{ \pm}\right] } & = \pm \breve{J}_{ \pm}, \quad\left[\breve{J}_{+}, \breve{J}_{-}\right]=-2 \mathcal{C}_{J J}\left(\breve{J}_{0}\right) \breve{J}_{0}
\end{aligned}
$$

The operator-valued coefficients

$$
\begin{aligned}
& \mathcal{C}_{\mathcal{L L}}\left(\breve{J}_{0}\right)=2\left(8 \breve{J}_{0}^{2}-10 \breve{J}_{0}+1\right), \\
& \mathcal{C}_{J \mathcal{L}}\left(\breve{J}_{0}\right)=16\left(\breve{J}_{0}-1\right)\left(3 \breve{J}_{0}-1\right), \\
& \mathcal{C}_{J J}\left(\breve{J}_{0}\right)=16\left(2 \breve{J}_{0}-1\right)\left(\breve{J}_{0}-1\right)\left(24 \breve{J}_{0}^{2}-14 \breve{J}_{0}+7\right)
\end{aligned}
$$

appear here instead of the unit coefficients in the $\mathfrak{o} \mathfrak{g} \mathfrak{p}(1 \mid 2)$ superalgebra (3.5), (3.6), and (3.7) of the QHO. The ground state $\Psi_{0}$ is annihilated by all the generators of the superalgebra and carries its trivial one-dimensional representation. On the higher bound states $\Psi_{n}, n=1,2, \ldots$, an infinite-dimensional irreducible representation of the superalgebra is realized. The structure with two irreducible representations is reflected coherently in the discrete flows of the ladder operators depicted in Fig. 3.

To conclude this section we note that the case of the deformed $\mathfrak{o} \mathfrak{g} \mathfrak{p}(1 \mid 2)$ superalgebra of the REQHO as well as the $\mathfrak{o} \mathfrak{s} \mathfrak{p}(1 \mid 2)$ Lie superalgebra of the QHO system can be considered as particular cases of the algebra generated by the three elements $h, \alpha^{+}$, and $\alpha^{-}$subject to the relations

$$
\left[h, \alpha^{ \pm}\right]= \pm 2 \alpha^{ \pm}, \quad\left\{\alpha^{+}, \alpha^{-}\right\}=F(h)+F(h+2),
$$

where $F(h)$ is some polynomial [37]. Such an algebra is characterized by the central element

$\Xi=\alpha^{+2} \alpha^{-2}+\alpha^{+} \alpha^{-}(F(h)-F(h-2))-(F(h))^{2}$.

In the case of the $\mathrm{QHO}$ we have the correspondences $\alpha^{ \pm}=a^{ \pm}, h=N+1$, and $F(h)=N$. The quadratic Casimir (3.9) of the Lie superalgebra $\mathfrak{o} \mathfrak{g} \mathfrak{p}(1 \mid 2)$ generated by the rescaled operators $\alpha^{ \pm}, \alpha^{ \pm 2}$, and $h$ is nothing but the central element $\Xi$ rescaled and shifted for an additive constant, $\mathcal{C}_{\mathfrak{p} \mathfrak{p} \mathfrak{p}(1 \mid 2)}=\frac{1}{16}(\Xi-1)$. For the REQHO system the operators $\alpha^{ \pm}$correspond to the ladder operators $\mathcal{A}^{ \pm}$, and we have $h=\breve{H}, F(h)=\Phi(\breve{H})$ with $\Phi(\breve{H})$ defined in Eq. (4.7). The superalgebra (4.23)-(4.25) in this case can be considered as a polynomial deformation of the $\mathfrak{o} \mathfrak{a p}(1 \mid 2)$ superalgebra. Using Eqs. (4.7) and (4.10), one can easily check that the central element $\Xi$ reduces identically to zero.

\section{CONCLUSION AND OUTLOOK}

To conclude, we list some problems that may be interesting for further investigation. 
We have constructed ladder operators for the simplest version of the REQHO system by the Darboux dressing of the creation and annihilation operators of the QHO. This was done by means of the first-order differential operators $A^{-}$and $A^{+}$which intertwine the REQHO and QHO Hamiltonians and factorize both of them. The procedure applied here is analogous to the procedure by which nontrivial Lax-Novikov integrals for reflectionless quantum systems are constructed by the Darboux dressing of the free particle's momentum operator [48]. But the same REQHO system can also be constructed by means of the Darboux-Crum-Krein-Adler procedure based on the use of several eigenstates of the QHO. In such a case the intertwiners will be higher-order differential operators. One can expect that the existence of different Darboux and Darboux-Crum-Krein-Adler transformations should reveal some new interesting aspects in the construction of the ladder operators for the REQHO and related dynamical symmetries (spectrum-generating algebras).

There exist other rational extensions of the QHO system. First, the analogs of the REQHO considered here can be generated by taking the nonphysical nodeless eigenstate $\psi_{2 n}^{-}$with $n>1$ as a function $\psi_{*}$ to generate the Darboux transformations. The ladder operators for such systems can be constructed in a similar way, by the Darboux dressing of the ladder operators of the QHO. We can also generate a corresponding polynomially deformed bosonized $\mathfrak{o} \mathfrak{s} \mathfrak{p}(1 \mid 2)$ superalgebra, whose trivial and infinite-dimensional representations will be realized on physical states of the corresponding rationally extended quantum harmonic oscillator. It would be interesting to see if there is any essential difference in the structure of the discrete flows generated by the ladder operators in such systems in comparison with the REQHO system considered here. The construction of the ladder operators by taking into account the existence of different Darboux-Crum-KreinAdler transformations to generate such systems should also reveal a dependence on the order of the polynomial that appears in the structure of the generating function $\psi_{2 n}^{-}(x)$ and on the size of the gap between the isolated ground state and the infinite tower of equidistant bound states.

A more complicated and rich picture from the point of view of the ladder operators and related symmetries can be expected in rationally extended quantum harmonic oscillator systems with a number $l>1$ of isolated bound states in the spectrum. There, a priori two essentially different cases should be distinguished. One case is when $l>1$ bound states will be separated from the infinite tower of equidistant bound states without any additional gaps between those $l$ states. Another, more general case is when isolated states include some additional gaps between themselves.

It is known that Jordan states appear in confluent Darboux-Crum transformations [44]. In particular, they were employed recently for the design of the $P T$-symmetric optical systems with invisible periodicity defects as well as completely invisible reflectionless $P T$-symmetric systems [43]. It would be interesting to look for possible physical applications of the generalized Jordan states considered here.

The considered REQHO system as well as its generalizations also seem to be interesting from the point of view of possible physical applications since-unlike other known deformations of the QHO, e.g., those related to the minimal length uncertainty relation $[49,50]$ - they provide a very specific change of the spectrum. Namely, they effectively add a finite number of bound states in the lower part of the QHO spectrum, separated by an additional (adjustable) gap, without disturbing the equidistant character of the rest of the infinite tower of the discrete levels. In this aspect they are very similar (as has been noted above in different but related context) to the quantum reflectionless systems which add a finite number of discrete bound states to the spectrum of the free particle. Such reflectionless systems are directly related to the soliton solutions to the Korteweg-de Vries and modified Korteweg-de Vries equations, and they find a lot of interesting applications in very diverse areas of physics including QCD, cosmology, solid states physics, the physics of polymers, plasma physics, and quantum optics, just to mention a few. ${ }^{2}$ Further results related to the ladder operators in rationally extended harmonic oscillator systems, which exploit the indicated similarity, will be presented elsewhere [52].

\section{ACKNOWLEDGMENTS}

M. S. P. thanks Y. Ispolatov for discussions. J. F. C. and M. S. P. acknowledge support from research projects FONDECYT 1130017 (Chile), Proyecto Basal USA1555 (Chile), MTM2015-64166-C2-1 (MINECO, Madrid), and DGA E24/1 (DGA, Zaragoza). M. S. P. is grateful for the warm hospitality at Zaragoza University. J. F. C. thanks for the kind hospitality at Universidad de Santiago de Chile.

\section{APPENDIX: DISCRETE CHAINS OF THE STATES OF THE QHO}

We describe here the action of the ladder operators $a^{+}$ and $a^{-}$on nonphysical eigenstates $\widetilde{\psi_{n}}$ and $\widetilde{\psi_{n}^{-}}$of the QHO and the construction of the associated Jordan and generalized Jordan states.

Making use of the identities $H_{n}^{\prime}=2 n H_{n-1}$ and $H_{n}=2 x H_{n-1}-H_{n-1}^{\prime}$ for Hermite polynomials, we obtain the relations

$$
\begin{aligned}
\int^{x} \frac{e^{\xi^{2}}}{H_{n}^{2}(\xi)} d \xi & =-\frac{1}{2 n} \int^{x} \frac{e^{\xi^{2}}}{H_{n-1}(\xi)} d\left(\frac{1}{H_{n}(\xi)}\right) \\
& =-\frac{e^{x^{2}}}{2 n H_{n} H_{n-1}}+\frac{1}{2 n} \int^{x} \frac{e^{\xi^{2}}}{H_{n-1}^{2}} d \xi
\end{aligned}
$$

\footnotetext{
${ }^{2}$ See, e.g., Refs. [15,48,51] and references therein.
} 
from which we find that

$$
a^{-} \widetilde{\psi_{n}}=\widetilde{\psi_{n-1}}, \quad n=1,2, \ldots
$$

Applying the operator $a^{+}$to both sides of this equality gives

$$
a^{+} \widetilde{\psi_{n}}=\widetilde{\psi_{n+1}}, \quad n=0,1, \ldots
$$

Changing $x \rightarrow i x$ in Eqs. (A2) and (A3), and taking into account that $a^{-} \rightarrow i a^{+}$, we also obtain

$$
\begin{aligned}
a^{+} \widetilde{\psi_{n}^{-}} & =\widetilde{\psi_{n-1}^{-}}, \quad n=1,2, \ldots, \quad a^{-} \widetilde{\psi_{n}^{-}}=\widetilde{\psi_{n+1}^{-}}, \\
n & =0,1, \ldots .
\end{aligned}
$$

In correspondence with Eqs. (2.5) and (2.6),

$$
a^{-} \widetilde{\psi_{0}}=\frac{1}{\psi_{0}}=\psi_{0}^{-}, \quad a^{+} \widetilde{\psi_{0}^{-}}=\psi_{0} .
$$

We also have

$\chi_{2}^{-}(x)=\psi_{0}(x) \int^{x} \psi_{0}^{-}(\xi) \widetilde{\psi_{0}^{-}}(\xi) d \xi, \quad a^{-} \chi_{2}^{-}(x)=\widetilde{\psi_{0}^{-}} \equiv \chi_{1}^{+}$.

This is a Jordan state which obeys the relations $(H-1) \chi_{2}^{-}=\psi_{0},(H-1)^{2} \chi_{2}^{-}=0$, where $H=a^{+} a^{-}+1$. Analogously,

$\chi_{2}^{+}(x)=\frac{1}{\psi_{0}} \int^{x} \psi_{0}(\xi) \widetilde{\psi_{0}}(\xi) d \xi, \quad a^{+} \chi_{2}^{+}=\widetilde{\psi_{0}} \equiv \chi_{1}^{-}$,

and $(H+1) \chi_{2}^{+}=\psi_{0}^{-},(H+1)^{2} \chi_{2}^{+}=0$.

Proceeding from the states $\chi_{2}^{+}$and $\chi_{2}^{-}$, one can construct an infinite net of Jordan and generalized Jordan states that are related to them. First, as analogs of $\Omega_{n}$ and $\breve{\Omega}_{n}$ defined in Eq. (2.10) we have the states $\chi_{n}^{-}$and $\chi_{n}^{+}$,

$$
\begin{aligned}
& \chi_{n}^{-}(x)=\psi_{0}(x) \int^{x} \psi_{0}^{-}(\xi) \chi_{n-1}^{+}(\xi) d \xi \\
& \chi_{n}^{+}(x)=\psi_{0}^{-}(x) \int^{x} \psi_{0}(\xi) \chi_{n-1}^{-}(\xi) d \xi
\end{aligned}
$$

where the case $n=1$ is also included by assuming $\chi_{0}^{-} \equiv \psi_{0}$ and $\chi_{0}^{+} \equiv \psi_{0}^{-}$. These are the higher-order Jordan states (2.10) generated on the basis of $\psi_{*}=\psi_{0}$. They satisfy the relations $a^{-} \chi_{n}^{-}=\chi_{n-1}^{+}, a^{+} \chi_{n}^{+}=\chi_{n-1}^{-}$, and, consequently, $a^{-}\left(a^{+} a^{-}\right)^{n} \chi_{2 n}^{-}=0, a^{+}\left(a^{-} a^{+}\right)^{n} \chi_{2 n}^{+}=0$. Therefore, $(H-1)^{n+1} \chi_{k}^{-}=0$ and $(H+1)^{n+1} \chi_{k}^{+}=0$ for $k=2 n, 2 n+1$.
One can define $\sigma_{n}^{-}=a^{+} \chi_{n}^{-}, \sigma_{n}^{+}=a^{-} \chi_{n}^{+}, n=2, \ldots$ These are Jordan states that obey the relations $(H-3)^{n} \sigma_{2 n}^{-}=\psi_{1},(H-3)^{n} \sigma_{2 n+1}^{-}=\widetilde{\psi_{1}},(H+3)^{n} \sigma_{2 n}^{+}=\psi_{1}^{-}$, $(H+3)^{n} \sigma_{2 n+1}^{+}=\widetilde{\psi_{1}^{-}}$, and, therefore, $(H-3)^{n+1} \sigma_{k}^{-}=0$, $(H+3)^{n+1} \sigma_{k}^{+}=0$ for $k=2 n, 2 n+1$.

In the same vein the family of Jordan states $\tau_{n}^{-}=a^{+} \sigma_{n}^{-}$ and $\tau_{n}^{+}=a^{-} \sigma_{n}^{+}, n=2, \ldots$ can be defined. They satisfy the relations $(H-5)^{n} \tau_{2 n}^{-}=\psi_{2},(H-5)^{n} \tau_{2 n+1}^{-}=\tilde{\psi}_{2},(H+$ $5)^{n} \tau_{2 n}^{+}=\psi_{2}^{-}, \quad(H+5)^{n} \tau_{2 n+1}^{+}=\widetilde{\psi_{2}^{-}}$, and $(H-5)^{n+1} \tau_{k}^{-}=0$, $(H+5)^{n+1} \tau_{k}^{+}=0$ for $k=2 n, 2 n+1$. These discrete flows can be further continued "horizontally."

On the other hand, the states defined via $\gamma_{n}^{-}=a^{+} \sigma_{n}^{-}$, $\gamma_{n}^{+}=a^{-} \sigma_{n}^{+}, n=2, \ldots$ are reduced to linear combinations of the already introduced Jordan states. Namely, $\gamma_{n}^{-}$is a linear combination of $\chi_{n}^{-}$and $\chi_{n-2}^{-}$, and $\gamma_{n}^{+}$is a linear combination of $\chi_{n}^{+}$and $\chi_{n-2}^{+}$.

Consider the states $\lambda_{n}^{ \pm}$given by means of the relations $a^{-} \lambda_{n}^{-}=\chi_{n-1}^{-}, a^{+} \lambda_{n}^{+}=\chi_{n-1}^{+}, n=3, \ldots$ The states $\lambda_{n}^{-}$can be presented in a form similar to that for $\chi_{n}^{-}$in Eq. (A8) but with $\chi_{n-1}^{+}$in the integrand changed for $\chi_{n-1}^{-}$. Analogously, $\lambda_{n}^{+}$are presented similarly to $\chi_{n}^{+}$in Eq. (A8) with $\chi_{n-1}^{-}$ in the integrand changed for $\chi_{n-1}^{+}$. For these states we have the relations $\left(a^{+} a^{-}\right)^{n} a^{-} \lambda_{2 n}^{-}=0,\left(a^{-} a^{+}\right)^{n} a^{+} \lambda_{2 n}^{+}=0$, $a^{-}\left(a^{+} a^{-}\right)^{n} a^{-} \lambda_{2 n+1}^{-}=0, a^{+}\left(a^{-} a^{+}\right)^{n} a^{+} \lambda_{2 n+1}^{+}=0$. As a consequence, $(H-1) \lambda_{n}^{-}=\chi_{n-2}^{+},(H+1) \lambda_{n}^{+}=\chi_{n-2}^{-}$. Therefore, these are generalized Jordan states which obey the relations $(H-1)(H-3)^{n} \lambda_{k}^{-}=0, \quad(H+1)(H+3)^{n} \lambda_{k}^{+}=0 \quad$ with $k=2 n, 2 n-1$.

Similarly, generalized Jordan states $\mu_{n}^{ \pm}(x)$ can be defined by proceeding from the states $\lambda_{n}^{ \pm}$via the relations $a^{+} \mu_{n}^{-}=\lambda_{n+1}^{-}, a^{-} \mu_{n}^{+}=\lambda_{n+1}^{+}, n=2, \ldots$ Then

$$
\begin{aligned}
& \mu_{n}^{-}(x)=\psi_{0}^{-}(x) \int^{x} \psi_{0}(\xi) \lambda_{n+1}^{-}(\xi) d \xi \\
& \mu_{n}^{+}(x)=\psi_{0}(x) \int^{x} \psi_{0}^{-}(\xi) \lambda_{n+1}^{+}(\xi) d \xi
\end{aligned}
$$

For these states we have $\chi_{n}^{-}=(H+1) \mu_{n}^{-}, \quad \chi_{n}^{+}=$ $(H-1) \mu_{n}^{+}$. They are generalized Jordan states that obey the relations $(H+1)(H-1)^{n+1} \mu_{k}^{-}=0, \quad(H-1)(H+$ $1)^{n+1} \mu_{k}^{+}=0$ with $k=2 n, 2 n+1$.

The described procedure of the construction of the Jordan and generalized Jordan states can be continued further in the obvious way. The discrete flows corresponding to the action of the ladder operators on the physical and associated nonphysical eigenstates of the QHO Hamiltonian and associated Jordan and generalized Jordan states are illustrated in Fig. 2. 
[1] G. Darboux, Sur une proposition relative aux équations linéaires, C.R. Hebd. Seances Acad. Sci. 94, 1456 (1882).

[2] M. M. Crum, Associated Sturm-Liouville systems, Q. J. Math. 6, 121 (1955).

[3] M. G. Krein, On a continuous analogue of a Christoffel formula from the theory of orthogonal polynomials, Dokl. Akad. Nauk SSSR 113, 970 (1957).

[4] V. E. Adler, A modification of Crum's method, Theor. Math. Phys. 101, 1381 (1994).

[5] V. B. Matveev and M. A. Salle, Darboux Transformations and Solitons (Springer, Berlin, 1991).

[6] E. Schrödinger, A method of determining quantummechanical eigenvalues and eigenfunctions, Proc. R. Irish Acad., Sect. A 46, 9 (1940).

[7] L. Infeld and T. E. Hull, The factorization method, Rev. Mod. Phys. 23, 21 (1951).

[8] J. F. Cariñena and A. Ramos, Riccati equation, factorization method and shape invariance, Rev. Math. Phys. 12, 1279 (2000).

[9] B. Mielnik and O. Rosas-Ortiz, Factorization: Little or great algorithm?, J. Phys. A 37, 10007 (2004).

[10] E. Witten, Dynamical breaking of supersymmetry, Nucl. Phys. B188, 513 (1981).

[11] E. Witten, Supersymmetry and Morse theory, J. Diff. Geom. 17, 661 (1982).

[12] F. Cooper, A. Khare, and U. Sukhatme, Supersymmetry and quantum mechanics, Phys. Rep. 251, 267 (1995).

[13] J.F. Cariñena, D. J. Fernández, and A. Ramos, Group theoretical approach to the intertwined Hamiltonians, Ann. Phys. (N.Y.) 292, 42 (2001).

[14] J. F. Cariñena and A. Ramos, Generalized BäcklundDarboux transformations in one-dimensional quantum mechanics, Int. J. Geom. Methods Mod. Phys. 05, 605 (2008).

[15] A. Arancibia and M. S. Plyushchay, Chiral asymmetry in propagation of soliton defects in crystalline backgrounds, Phys. Rev. D 92, 105009 (2015).

[16] A. P. Veselov and A. B. Shabat, Dressing chains and the spectral theory of the Schrödinger operator, Funct. Anal. Appl. 27, 81 (1993).

[17] S. P. Novikov, S. V. Manakov, L. P. Pitaevskii, and V. E. Zakharov, Theory of Solitons (Plenum, New York, 1984).

[18] F. Gesztesy and H. Holden, Soliton Equations and their Algebro-Geometric Solutions (Cambridge University Press, Cambridge, England, 2003).

[19] V.E. Adler, Nonlinear chains and Painlevé equations, Physica (Amsterdam) 73D, 335 (1994).

[20] R. Willox and J. Hietarinta, Painlevé equations from Darboux chains: I. PIII—PV, J. Phys. A 36, 10615 (2003).

[21] J. J. Duistermaat and F. A. Grünbaum, Differential equations in the spectral parameter, Commun. Math. Phys. 103, 177 (1986).

[22] A. Oblomkov, Monodromy-free Schrödinger operators with quadratically increasing potentials, Theor. Math. Phys. 121, 1574 (1999).

[23] S. Yu. Dubov, V. M. Eleonskii, and N. E. Kulagin, Equidistant spectra of anharmonic oscillators, Zh. Eksp. Teor. Fiz. 102, 814 (1992) [Sov. Phys. JETP 75, 446 (1992)];S. Yu. Dubov, V. M. Eleonskii, and N. E. Kulagin, Chaos 4, 47 (1994).

[24] J. F. Cariñena, A. M. Perelomov, M. F. Rañada, and M. Santander, A quantum exactly solvable nonlinear oscillator related to the isotonic oscillator, J. Phys. A 41, 085301 (2008).

[25] J. M. Fellows and R. A. Smith, Factorization solution of a family of quantum nonlinear oscillators, J. Phys. A 42, 335303 (2009).

[26] D. Gómez-Ullate, N. Kamran, and R. Milson, An extended class of orthogonal polynomials defined by a SturmLiouville problem, J. Math. Anal. Appl. 359, 352 (2009); An extension of Bochner's problem: exceptional invariant subspaces, J. Approx. Theory 162, 987 (2010).

[27] S. Odake and R. Sasaki, Infinitely many shape invariant potentials and new orthogonal polynomials, Phys. Lett. B 679, 414 (2009); Another set of infinitely many exceptional (X) Laguerre polynomials, Phys. Lett. B 684, 173 (2010).

[28] R. Sasaki, S. Tsujimoto, and A. Zhedanov, Exceptional Laguerre and Jacobi polynomials and the corresponding potentials through Darboux-Crum transformations, J. Phys. A 43, 315204 (2010).

[29] C. Quesne, Exceptional orthogonal polynomials, exactly solvable potentials and supersymmetry, J. Phys. A 41, 392001 (2008); Solvable rational potentials and exceptional orthogonal polynomials in supersymmetric quantum mechanics, SIGMA 5, 084 (2009).

[30] Y. Grandati, Solvable rational extensions of the isotonic oscillator, Ann. Phys. (Amsterdam) 326, 2074 (2011).

[31] J. Sesma, The generalized quantum isotonic oscillator, J. Phys. A 43, 185303 (2010).

[32] D. Gómez-Ullate, Y. Grandati, and R. Milson, Rational extensions of the quantum harmonic oscillator and exceptional Hermite polynomials, J. Phys. A 47, 015203 (2014).

[33] A. M. Pupasov-Maksimov, Propagators of isochronous anharmonic oscillators and Mehler formula for the exceptional Hermite polynomials, Ann. Phys. (Amsterdam) 363, 122 (2015)

[34] M. Asorey, J. F. Carinena, G. Marmo, and A. Perelomov, Isoperiodic classical systems and their quantum counterparts, Ann. Phys. (Amsterdam) 322, 1444 (2007).

[35] J. F. Cariñena, A. M. Perelomov, and M. F. Rañada, Isochronous classical systems and quantum systems with equally spaced spectra, J. Phys. Conf. Ser. 87, 012007 (2007).

[36] M. de Crombrugghe and V. Rittenberg, Supersymmetric quantum mechanics, Ann. Phys. (N.Y.) 151, 99 (1983).

[37] J. Van der Jeugt and R. Jagannathan, Polynomial deformations of $\operatorname{osp}(1 / 2)$ and generalized parabosons, J. Math. Phys. 36, 4507 (1995).

[38] S. Krivonos and O. Lechtenfeld, $S U(2)$ reduction in $\mathcal{N}=4$ supersymmetric mechanics, Phys. Rev. D 80, 045019 (2009).

[39] S. Fedoruk and J. Lukierski, Algebraic structure of Galilean superconformal symmetries, Phys. Rev. D 84, 065002 (2011).

[40] M. S. Plyushchay and A. Wipf, Particle in a self-dual dyon background: Hidden free nature, and exotic superconformal symmetry, Phys. Rev. D 89, 045017 (2014).

[41] G. F. de Téramond, H. G. Dosch, and S. J. Brodsky, Baryon spectrum from superconformal quantum mechanics and its light-front holographic embedding, Phys. Rev. D 91, 045040 (2015). 
[42] E. Ivanov, S. Sidorov, and F. Toppan, Superconformal mechanics in $S U(2 \mid 1)$ superspace, Phys. Rev. D 91, 085032 (2015).

[43] F. Correa, V. Jakubsky, and M. S. Plyushchay, PTsymmetric invisible defects and confluent Darboux-Crum transformations, Phys. Rev. A 92, 023839 (2015).

[44] A. Schulze-Halberg, Wronskian representation for confluent supersymmetric transformation chains of arbitrary order, Eur. Phys. J. Plus 128, 68 (2013).

[45] M. S. Plyushchay, Deformed Heisenberg algebra, fractional spin fields and supersymmetry without fermions, Ann. Phys. (N.Y.) 245, 339 (1996); Hidden nonlinear supersymmetries in pure parabosonic systems, Int. J. Mod. Phys. A 15, 3679 (2000); V. Jakubsky, L. M. Nieto, and M. S. Plyushchay, The origin of the hidden supersymmetry, Phys. Lett. B 692, 51 (2010).

[46] S. Post, L. Vinet, and A. Zhedanov, Supersymmetric quantum mechanics with reflections, J. Phys. A 44, 435301 (2011); V. X. Genest, L. Vinet, G.-F. Yu, and A. Zhedanov, Supersymmetry of the quantum rotor, arXiv: 1607.06967.

[47] F. Correa and M. S. Plyushchay, Hidden supersymmetry in quantum bosonic systems, Ann. Phys. (Amsterdam)
322, 2493 (2007); F. Correa, L. M. Nieto, and M. S. Plyushchay, Hidden nonlinear supersymmetry of finitegap Lamé equation, Phys. Lett. B 644, 94 (2007); F. Correa, V. Jakubsky, L. M. Nieto, and M. S. Plyushchay, Self-Isospectrality, Special Supersymmetry, and their Effect on the Band Structure, Phys. Rev. Lett. 101, 030403 (2008).

[48] A. Arancibia, J. Mateos Guilarte, and M. S. Plyushchay, Effect of scalings and translations on the supersymmetric quantum-mechanical structure of soliton systems, Phys. Rev. D 87, 045009 (2013).

[49] A. Kempf, Uncertainty relation in quantum mechanics with quantum group symmetry, J. Math. Phys. 35, 4483 (1994); A. Kempf, G. Mangano, and R. B. Mann, Hilbert space representation of the minimal length uncertainty relation, Phys. Rev. D 52, 1108 (1995).

[50] M. A. C. Rossi, T. Giani, and M. G. A. Paris, Probing deformed quantum commutators, Phys. Rev. D 94, 024014 (2016).

[51] A. Arancibia and M.S. Plyushchay, Transmutations of supersymmetry through soliton scattering and selfconsistent condensates, Phys. Rev. D 90, 025008 (2014).

[52] J. F. Cariñena and M. S. Plyushchay (to be published). 